ticular, they often show semi-classical characteristics that simplify the analysis of the cross-sections, and also the heavy ions bring in high angular momenta that make it possible to excite states of high spin. Furthermore, these reactions often show marked selectivity in that they tend preferentially to populate states of a particularly simple structure.

The distorted wave Born approximation (DWBA) formalism has been used with great success to analyse the $(d, p)$ and similar reactions; it has yielded important information on nuclear structure, particularly the spectroscopic factors. This theory has naturally also been applied to one-nucleon transfer reactions between heavy ions and it has been found to give excellent results in some cases but not in others.

A particularly notable discrepancy is found for reactions between light nuclei. These have cross-sections that fall off steadily with increasing angle, whereas the DWBA predicts angular distributions showing a marked oscillatory structure. The reason for this is that in conventional DWBA calculations the nuclear recoil is neglected, and this is justified if the mass of the incident particle is much less than that of the target nucleus. In the case of reactions between heavy ions these masses are comparable and recoil can no longer be ignored.

Examination of the angular momentum conservation relations in the reactions shows that if recoil is neglected

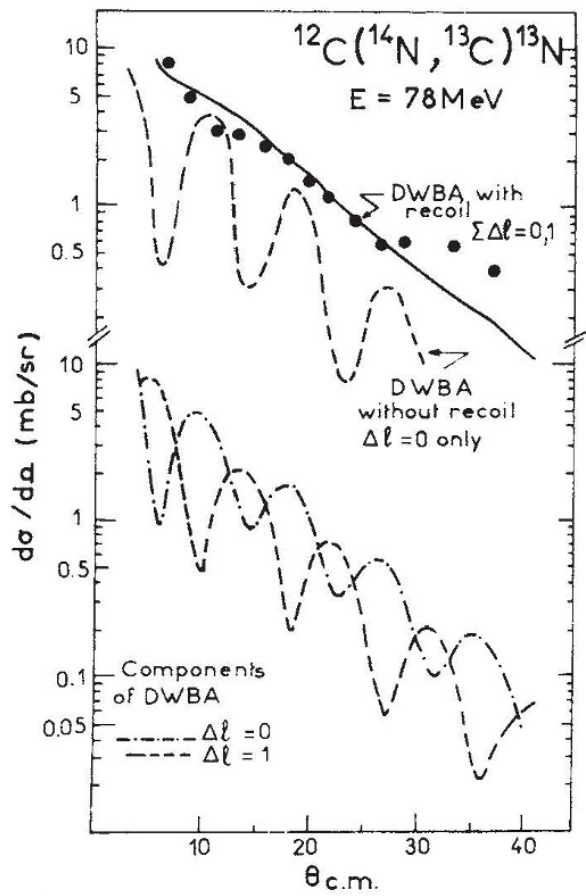

Differential cross-section for a onenucleon heavy ion transfer reaction compared with DWBA calculations. The upper dashed curve shows the DWBA calculations without recoil $(\Delta l=0$ only) and the two lower curves the DWBA calculations with recoil $(\Delta l=0$ and 1$)$. The sum of these two lower curves is the full curve, which agree well with the experimental data. the change $\Delta l$ in the orbital angular momentum of the transferred particle must be zero, whereas if recoil is taken into account $\Delta l$ may be 0 or 1 .

The inclusion of recoil effects makes the calculations much more complicated, but it still remains within the capacity of fast computers. A program including recoil effects has recently been written and DeVries and Kubo (Phys. Rev. Lett., 30, 325 ; 1973) have used it to investigate the importance of these effects. They find, as shown in the diagram, that the $\Delta l=0$ and $\Delta l=1$ contributions to the reaction amplitude both oscillate as a function of reaction angle, but that the oscillations are out of phase so that their sum varies smoothly with angle, just like the experimental data.
This shows very clearly that recoil effects must be included in DWBA calculations of nucleon transfer between heavy ions of comparable mass.

The calculations also give the product of the two spectroscopic factors for the transition, and this is close to the theoretical value of Cohen and Kurath. Thus the DWBA theory is able to give a good account of both the angular distribution and the absolute magnitude of the cross-section for single-nucleon transfer between heavy ions. It now seems likely, subject to further tests, that these reactions can be used with some confidence to determine unknown spectroscopic factors and will thus become an important tool for the determination of nuclear structure.

\title{
Ulitrusonic Velocities in Troodos (Cyprus) Rocks
}

Although the idea that the Troodos Igneous Complex of Cyprus is an upthrust segment of (Mesozoic) seafloor has been around now for about a decade, the evidence to settle the matter with certainty has still not been obtained. Nevertheless, most, if not all, of the relevant geophysical data currently available are consistent with the seafloor interpretation, suggesting that any refutation of the idea in the future is unlikely.

In the meantime, the search for that vital piece of evidence goes on, largely by geophysical means. One of the obvious approaches to the problem is the measurement of seismic velocities, the hope being that the velocity structure of the Troodos Massif will prove to correspond with the unique structure of the present ocean floor rather than with the variable structures of continents. Matthews et al. (Nature Physical Science, 231, 200 ; 1971), Lort and Matthews (Geophys. J., 27, 383; 1972) and Khan et al. (Nature Physical Science, 238, $134 ;$ 1972) have all made seismic determinations in the field; but through no fault of these workers, all these investigations have been less than ideal. One of the principal difficulties is that, being upthrust, the Troodos Complex is no longer subject to the confining pressures typical of present or ancient ocean floor in situ, so that seismic velocities can hardly be expected to correspond directly. In addition, fracturing in the Troodos units has almost certainly reduced seismic velocities from those applicable at ocean floor depths.

In next Monday's Nature Physical Science (May 7), Poster reports attempts to solve these problems by measuring velocities in Troodos samples under pressure in the laboratory. He has determined ultrasonic velocities under hydrostatic pressures of up to $2 \mathrm{kbar}$ and at room temperature for nineteen rock samples representative of each of the principal Troodos units. Typically, velocities increase rapidly up to pressures of about $1 \mathrm{kbar}$ and thereafter remain relatively constant, the initial increase being most marked for the pillow lavas and rocks from the sheeted intrusive complex. But even at atmospheric pressure, velocities were higher than those measured in the field, presumably because of the relative lack of fracturing in the smaller laboratory samples. This velocity difference was greatest in rocks of the plutonic complex (gabbro and ultramafics).

With the exception of the ultramafics, the velocities measured in the laboratory increased with downward stratigraphic order, encouraging Poster to compare the velocity structure with that of "normal" oceanic crust (assuming a purely confining pressure to be exerted on the top of layer 2). At the relevant pressures of $0.5-1.0 \mathrm{kbar}$, the pillow lava velocities were lower than, and the velocities in the sheeted intrusive complex were generally higher than, the average for oceanic layer $2\left(5.1 \mathrm{~km} \mathrm{~s}^{-1}\right)$. Thus the basal group of the sheeted intrusive complex, comprising screens of pillow lavas with diabase dykes and thought to correspond with layer 2, could easily produce the mean velocity of layer 2 and the wide range of velocities observed in it. Stratigraphically below, measured velocities then increased progressively to typical layer 3 velocities for the fresh gabbros.

The new velocity data thus generally support the view that the Troodos Complex began as oceanic crust, the only anomaly being the fact that the ultramafics have lower velocities at all pressures than the gabbros which lie stratigraphically above. Poster's explanation for this discrepancy is based on his discovery that seven of the ten ultramafics examined were at least 75 per cent serpentinized. 